\title{
BMJ Open The costs of functional gastrointestinal disorders and related signs and symptoms in infants: a systematic literature review and cost calculation for England
}

James Mahon, ${ }^{1}$ Carlos Lifschitz, ${ }^{2}$ Thomas Ludwig, ${ }^{3}$ Nikhil Thapar, ${ }^{4}$ Julie Glanville, ${ }^{1}$ Mohamad Miqdady, ${ }^{5}$ Miguel Saps, ${ }^{6}$ Seng Hock Quak, ${ }^{7}$ Irene Lenoir Wijnkoop, ${ }^{8}$ Mary Edwards, ${ }^{1}$ Hannah Wood, ${ }^{1}$ Hania Szajewska ${ }^{9}$

To cite: Mahon J, Lifschitz C, Ludwig T, et al. The costs of functional gastrointestinal disorders and related signs and symptoms in infants: a systematic literature review and cost calculation for England. BMJ Open 2017;7:e015594. doi:10.1136/ bmjopen-2016-015594

- Prepublication history and additional material for this paper are available online. To view these files, please visit the journal online (http://dx.doi org/10.1136/bmjopen-2016015594).

Received 15 December 2016 Revised 20 October 2017 Accepted 23 0ctober 2017

CrossMark

For numbered affiliations see end of article.

Correspondence to Dr Carlos Lifschitz; carlos.lifschitz@hospitalitaliano. org.ar

\section{ABSTRACT}

Objectives To estimate the cost of functional gastrointestinal disorders (FGIDs) and related signs and symptoms in infants to the third party payer and to parents.

Study design To estimate the cost of illness (COI) of infant FGIDs, a two-stage process was applied: a systematic literature review and a COI calculation. As no pertinent papers were found in the systematic literature review, a 'de novo' analysis was performed. For the latter, the potential costs for the third party payer (the National Health Service (NHS) in England) and for parents/carers for the treatment of FGIDs in infants were calculated, by using publicly available data. In constructing the calculation, estimates and assumptions (where necessary) were chosen to provide a lower bound (minimum) of the potential overall cost. In doing so, the interpretation of the calculation is that the true COI can be no lower than that estimated.

Results Our calculation estimated that the total costs of treating FGIDs in infants in England were at least $£ 72.3$ million per year in $2014 / 2015$ of which $£ 49.1$ million was NHS expenditure on prescriptions, community care and hospital treatment. Parents incurred £23.2 million in costs through purchase of over the counter remedies. Conclusions The total cost presented here is likely to be a significant underestimate as only lower bound estimates were used where applicable, and for example, costs of alternative therapies, inpatient treatments or diagnostic tests, and time off work by parents could not be adequately estimated and were omitted from the calculation. The number and kind of prescribed products and products sold over the counter to treat FGIDs suggest that there are gaps between treatment guidelines, which emphasise parental reassurance and nutritional advice, and their implementation.

\section{INTRODUCTION}

Functional gastrointestinal disorders (FGIDs), according to Rome IV criteria, are defined as variable combinations of chronic

\section{Strengths and limitations of the study}

- The cost calculation is focused on more recent studies and data to ensure currency and most recent practice are reflected in terms of care of FGIDs and related signs and symptoms.

- Where necessary, estimates and assumptions were always chosen to provide consistently a lower bound of the potential cost.

- The total cost presented here is likely to be a significant underestimate of the true cost as lower bound estimates were used where applicable, and several costs could not be adequately estimated and were omitted from the calculation.

or recurrent gastrointestinal (GI) signs and symptoms without obvious structural or biochemical alterations. ${ }^{1}$ Within the first year after birth, such symptoms can be observed in up to $50 \%$ of infants. ${ }^{23}$

A recent meta-review reported that the worldwide prevalence of the three most common FGIDs in infants, infantile regurgitation, colic and functional constipation, is approximately $30 \%, 20 \%$ and $15 \%$, respectively. ${ }^{4}$ In addition, many children may present with a combination of FGIDs and related signs and symptoms. ${ }^{34}$ Although considered mostly as benign conditions, FGIDs are a source of concern and frustration for families that may cause them to seek the advice from healthcare professionals (HCPs). ${ }^{34}$

Diagnostic criteria for FGIDs have been defined and are being continuously revised, and algorithms have been developed for their practical management by HCPs. ${ }^{1-6}$ These algorithms build on parental support, reassurance and nutritional advice as first-line therapy. Depending on the specific condition, 
advice is given on issues including feeding frequency and volume as well as allergen avoidance in both breast and formula fed infants. Despite stringent diagnostic criteria and treatment recommendations, daily practices may broadly deviate from these and infants suffering from FGIDs and related signs and symptoms receive a large number of other treatments that are either contraindicated or not substantiated scientifically. ${ }^{7}$

The aim of this study was to estimate the cost of FGIDs and related signs and symptoms in infants to the third party payer and to parents.

\section{METHODS}

The study employed a two-stage methodology to estimate the cost of illness (COI) of infant FGIDs, a systematic literature review and a COI calculation. Here, we report in detail on the latter.

\section{Stage 1}

A systematic literature review was undertaken to identify any studies published in or after 2005 that provided information on (a) the frequency and volume of reported treatments of FGIDs and related signs and symptoms (regardless of their effectiveness); (b) costs to third party payers and/or parents of infants with FGIDs and related signs and symptoms of prescribed treatments, over the counter (OTC) or home remedies, visits to HCPs and other providers of complementary and other forms of care, and changes in infant formula; (c) loss of income for parents/carers of infants with FGIDs and related signs and symptoms, or the specific symptom combinations described above, through inability to return to work, time taken off work and out of pocket costs.

Studies of infants $<12$ months old with colic, regurgitation and/or functional constipation were eligible for inclusion if the underlying cause of illness was believed to be related to a FGID. Studies in preterm infants were excluded. The details of the review methods and protocol have been published in detail. ${ }^{8}$ Studies reporting data about treatments, signs and symptoms of FGIDs were considered separately to studies reporting direct and indirect costs.

\section{Stage 2: COI calculation for one country}

Since the systematic review identified no research on COI for any country, we performed a calculation for one country using evidence from stage 1 (the literature review) where appropriate, and from readily available data sources. England was chosen as an exemplar country due to the availability and quality of data on healthcare resource use, both publicly and privately, and the availability of these data in the English language.

Potential costs were considered for the third party payer (the National Health Service (NHS) in England) and for parents/carers. In constructing the calculation, estimates and assumptions (where necessary) were chosen to provide a lower bound (minimum) of the potential overall cost. In doing so, the interpretation of the calculation is that the true COI can be no lower than that estimated.

\section{Publicly funded healthcare resource use Prescription data}

Potential medicinal remedies for infant FGIDs and special infant formulas were identified either through the systematic review or via clinical expert opinion. The prescribed items considered in the analysis with the number and costs of prescriptions made in England are available from the Health and Social Care Information Centre (HSCIC). Data were available for 2014/2015 and cover prescriptions made in both primary and secondary care.

Although the prescription analysis is precise on the cost of medications and formula, the analysis is not clear in all cases about whether the medicine or formula was indicated specifically for infants with FGIDs or specifically for those aged $<12$ months. Therefore, we made some assumptions. We assumed the colic remedies would be for children $<12$ months of age. If colicky symptoms had not cleared by this time, further investigations would be undertaken and it is difficult to envisage situations where a persistently crying baby who appeared in pain would still be prescribed medications that must have proven ineffective up to that point. In addition, the Rome III criteria for infantile colic include only children who are $<4$ months, although it is not certain that this, in itself, would stop a general practitioner (GP) prescribing colic remedies once an infant reached that age.

For gastro-oesophageal reflux, the combination of aluminium hydroxide and magnesium carbonate (Gaviscon infant) is suitable up to 24 months of age. However, clinical advice and evidence from systematic reviews suggest that nearly all reflux and regurgitation would clear by the age of 12 months. Hence, we estimated that $90 \%$ of the Gaviscon infant would be prescribed to children $<12$ months.

Proton pump inhibitors (PPIs) have not been included in the analysis as these should only be used in diagnosed gastro-oesophageal reflux disease which is not a FGID. However, PIPs have been reported to be overprescribed by paediatricians in general, and more specifically for infantile colic, though these have been proven to be ineffective $^{9}$ and have frequent side effects. ${ }^{10-12}$

For constipation, docusate sodium (Ducosol paediatric) is suitable for those up to the age of 12 years. Hence, we have divided the number of prescriptions and the cost by 12 to provide an estimate of prescriptions to those $<12$ months. Infant glycerol suppositories were also included, and we assumed that all prescriptions were for infants $<12$ months, because a paediatric formulation is available for those $>12$ months. We considered prescriptions of lactulose, but it was not possible to isolate a preparation just for infants and children. Most preparations for the treatment of constipation are not recommended for those $<12$ months of age even if, in practice, they may be used with infants. 


\section{Primary and community care costs}

From a community care perspective, an assumption was made that infants with infantile colic will require one extra home visit from a health visitor compared with babies without colic. Evidence suggests that the incidence of infantile colic is between $10 \%$ and $40 \% .{ }^{13} \mathrm{~A}$ National Institute of Health Research funded ongoing trial of supporting parents of infants with colic indicates an incidence of one in five infants. ${ }^{14}$ Applying the 1:5 figure to the 697852 infants born live in England and Wales in 2015 means that approximately 140000 infants in England experienced colic in that year.

Without data on the number of GP appointments, it has been assumed that as prescriptions will in most cases have been written by a GP, the number of appointments must, as a minimum, be equal to the number of prescriptions written. Although it is possible that more than one item could have been written at the same time, it was considered that in routine clinical practice for infantile colic, only one medicine would have been tried at any one time. Follow-up consultations have not been included in the analysis nor have any consultations that resulted in no prescription. As such, the estimate that GP consultations will be equal to the number of prescriptions will result in a conservative estimate of the true impact of GP time spent dealing with FGIDs.

\section{Hospital care}

Data on hospital care and activity are collected in England by each hospital and collated each year as the Hospital Episode Statistics (HES) dataset, available through the HSCIC. This dataset contains information on all accident and emergency (A\&E) and outpatient attendances and admitted patient care in England.

The admitted patient care dataset provides information on all planned and unplanned hospital admissions, including those seen as day cases. Planned admissions are usually for surgical procedures. Unplanned admissions can be for emergency operations but can also be for patients staying in hospital for observation. Data are available on the primary International Classification of Diseases 10th Revision (ICD-10) diagnostic code of the admitted patient as well as the age of the patient. We received expert advice on the ICD-10 codes that would be used exclusively for infant FGIDs. We excluded codes that could also be used for other conditions, resulting in our estimate being a lower bound of actual admissions for FGIDs.

HES collates data on all patients who present at hospital emergency rooms (A\&E in the UK). Data are not as detailed as those for admitted patient care, although age is recorded and along with a broad diagnosis group, but no ICD-10 code. Data by age and diagnosis jointly are not readily available.

Data are available from HES on outpatient appointments. Outpatient appointments in the UK usually relate to appointments with hospital-based consultants or diagnostic professionals, or in some cases to receive a simple treatment that does not require a hospital bed. Outpatient appointments are, in almost all cases, made through GP referral. A patient in England cannot in most cases access specialist treatment or diagnostic procedures without a GP referral unless they pay privately. Outpatient data are available by ICD-10 code, but not routinely broken down by age.

\section{OTC colic remedies and special infant formulas}

Data were provided by IRi (Information Resources, INC) on OTC sales of colic remedies, simethicone, lactase, various gripe waters and special infant formulas for the period 2014/2015.

\section{RESULTS}

\section{Stage 1: systematic review}

The full review results are presented in a supplement to this manuscript (online supplementary file). In total, 12364 records were identified from database searching and 78 from additional resources. After the steps of duplicate removal, title, abstract and full text screen, 31 studies were identified that provided data about treatments, signs and symptoms of FGID in infants. Three further studies provided additional data on young children in the USA. ${ }^{15-17}$ Twenty-six of the 31 eligible studies were randomised controlled trials and 5 were case series. ${ }^{8}$ Almost half (15) of these studies were undertaken in Europe $^{18-32}$ (including 3 in the UK). ${ }^{30-32}$ Four studies were conducted in the USA, ${ }^{33-36}$ three in Australia, ${ }^{37-39}$ three in Turkey ${ }^{40-42}$ and one each in Brazil, ${ }^{43}$ Canada, ${ }^{44}$ China, ${ }^{45}$ Iran, ${ }^{46}$ Israel ${ }^{12}$ and Nigeria. ${ }^{47}$ Twenty-nine studies included infants with infantile colic and two studies included infants with constipation. Several different interventions were addressed in the eligible studies. We could not identify any study that addressed the whole spectrum of costs of treating FGID in infants.

\section{Stage 2: COI calculation for England \\ Prescription data}

Medicines or formulas prescribed in England to infants are fully covered by the NHS. A full list of the prescribed items considered, the number of prescriptions and the associated cost is shown in table 1 .

We estimated the total number of prescriptions of colic and FGID medications for infants $<12$ months in $2014 / 2015$ to be 521000 , at a cost of $£ 5.8$ million and the total number of prescriptions of colic and antireflux formulas to be 58000 at a cost of $£ 0.9$ million.

\section{Primary and community care costs}

We estimated that the average time for a home visit, including travel, would be $30 \mathrm{~min}$, with a unit cost per half hour of $£ 25^{48}$ giving a cost of $£ 3.5$ million.

Data from table 1 for colic and FGID medicines and formulas suggested a total of 578000 prescriptions. At a cost of $£ 45$ per 11.7 min appointment, this would equate to a cost to the NHS of $£ 26.0$ million. $^{48}$ 
Table 1 Prescription analysis 2014/2015

\begin{tabular}{lcc}
\hline Type of solution & Sum of items (thousands) & Cost £ (millions) \\
\hline Medicinal & 521.2 & 5.8 \\
\hline Colic & 115.1 & 1.1 \\
$\quad$ Colief_Infant Dps & 64.7 & 0.9 \\
\hline Dentinox_Infant Colic Dps & 3.1 & $<0.1$ \\
Infacol_Susp 40 mg/mL S/F & 47.1 & 0.2 \\
\hline Nurse Harveys_Gripe Mix & $<0.1$ & $<0.1$ \\
Woodward's_Gripe Water & 0.2 & $<0.1$ \\
\hline Constipation & 24.8 & $<0.1$ \\
$\quad$ Glycerol Suppository Infants (1 g) & 23.9 & $<0.1$ \\
Docusol_Paed Soln 12.5 mg/5 ml S/F (1/12 of total prescriptions) & 0.9 & $<0.1$ \\
\hline Reflux and regurgitation & 381.4 & 4.7 \\
\hline Gaviscon Infant_Sach 2g (dual pack) S/F (9/10 of total prescriptions) & 381.4 & 4.7 \\
\hline Colic and regurgitation formulas & 58.8 & 0.9 \\
\hline Reflux and regurgitation & 55.8 & 0.8 \\
\hline Colic & 3.0 & 0.1 \\
\hline Grand total & 580.0 & 6.7 \\
\hline
\end{tabular}

Hospital care: admitted patient care

The total number of admissions for each of the ICD-10 diagnosis codes for FGIDs or colic, with the length of stay included in the analysis, are shown in table 2.

A total of 16183 infants were admitted to acute hospitals in 2014/2015 in England due to FGIDs amounting to 25800 bed days. The cost to the NHS of a day in a hospital bed in 2014/2015 was £359.13. ${ }^{49}$ The total cost of the admitted patient care was therefore $£ 9.3$ million. This

Table 2 Number of admissions and mean length of stay for patients with FGID or colic in England 2014/2015

\begin{tabular}{|c|c|c|c|}
\hline $\begin{array}{l}\text { ICD-10 } \\
\text { code }\end{array}$ & Description & $\begin{array}{l}\text { Number of } \\
\text { admissions }\end{array}$ & $\begin{array}{l}\text { Mean } \\
\text { length of } \\
\text { stay }\end{array}$ \\
\hline k21.9 & Reflux & 6717 & 1 \\
\hline p92.1 & $\begin{array}{l}\text { Regurgitation and } \\
\text { rumination in newborn }\end{array}$ & 136 & 1 \\
\hline f98.2 & $\begin{array}{l}\text { Feeding disorder of } \\
\text { infancy and childhood }\end{array}$ & 4 & 11 \\
\hline r11.1 & Vomiting & 4313 & 2 \\
\hline r10.4 & Colic & 885 & 1 \\
\hline k59.0 & $\begin{array}{l}\text { Constipation } \\
\text { (unspecified) }\end{array}$ & 2471 & 3 \\
\hline k59.1 & Functional diarrhoea & 5 & 6 \\
\hline r68.1 & $\begin{array}{l}\text { Excessive crying/fussy } \\
\text { infant }\end{array}$ & 1355 & 1 \\
\hline$r 14$ & $\begin{array}{l}\text { Flatulence and related } \\
\text { conditions }\end{array}$ & 297 & 2 \\
\hline
\end{tabular}

FGID, functional gastrointestinal disorder; ICD-10, International Classification of Diseases 10th Revision. cost is only for bed days and does not include the cost of any diagnostic procedures.

Hospital care: A\&E visits

The number of A\&E attendances for children $<12$ months of age was 483000 in 2014/2015 and the percentage of all attendances for all ages for all GI conditions was $5.7 \% .^{50}$ We estimated the number of attendances due to GI conditions in infants aged $<1$ year by assuming that the proportion of attendances due to GI conditions is the same across age groups. Evidence from the USA identified in the literature review suggested that $9.4 \%$ of all emergency department visits in the USA due to constipation were in those aged $<12$ months. ${ }^{16}$ If a similar pattern is seen in England and for all FGIDs, then this means that the estimated attendances we have calculated are likely to be a significant underestimate.

The reference cost of a NHS A\&E visit in 2014/2015 was $£ 132 .{ }^{49}$ So the total cost of all visits for infants in 2014/2015 was $£ 63.7$ million. If all these visits by infants were due to FGIDs then this is the upper bound of what the cost of A\&E services due to FGIDs could be. If the percentage attending A\&E due to GI conditions is the same regardless of age, this suggests that the cost of these infant visits is no higher than $£ 3.6$ million.

Hospital care: outpatient data

The total number of outpatient appointments for the conditions of interest in 2014/2015 was very small and were in single figures in some cases. For the two conditions with the highest number of appointments-constipation and reflux - there were 4000 episodes for all ages. Therefore, the number of appointments for children $<1$ year of age would potentially be insignificant, from 
a cost perspective. However, in $95.5 \%$ of outpatient appointments, the condition is recorded as unknown or unspecified. Costs associated with outpatient care were excluded from the analysis because we were unable to isolate the appointments from the dataset. Given there were $85.6 \mathrm{~m}$ outpatient appointments in England in $2014 / 2015$, if only a small percentage of these were for infants with FGIDs, the total costs would be substantial. The exclusion of these appointments from the analysis is, therefore, a further conservative element of the overall calculation.

\section{Alternative therapies}

The literature review highlighted that a range of alternative therapies, particularly for infantile colic, had been considered across many countries. Such therapies include chiropractic services, physiotherapy, homoeopathy, osteopathy and acupuncture. ${ }^{24} 27283031$ No data were identified in the literature on the scale of use of these therapies. We contacted professional associations and regulatory bodies associated with each therapy to request any data they might hold on this issue. However, none were able to provide information for the analysis. The costs of these approaches are therefore not stated, which constitutes an underestimate of the real costs.

\section{OTC colic remedies and special infant formulas}

The total expenditure on colic remedies was £13.6 million and on antiregurgitation formulas was $£ 9.6$ million.

\section{Estimated total cost infant FGIDs in England}

Combining the different aspects of publicly funded and parental out of pocket expenditure on infant FGIDs described above, we reached an overall estimate of the COI of the conditions in England in 2014/2015. This is summarised in table 3. In total, the cost is estimated to have been $£ 72.3$ million.

\section{Table 3 Summary of costs of colic/FGID in England} 2014/2015

\begin{tabular}{lc}
\hline Cost area & Value (million) \\
\hline $\begin{array}{l}\text { Prescriptions of colic/reflux/constipation } \\
\text { medicines }\end{array}$ & $£ 5.8$ \\
$\begin{array}{l}\text { Prescriptions of colic/reflux/constipation } \\
\text { formulas }\end{array}$ & $£ 0.9$ \\
$\begin{array}{lr}\text { Health visitor appointments } \\
\text { GP appointments (colic/reflux/constipation }\end{array}$ & $£ 26.0$ \\
medicines and formula) & $£ 9.3$ \\
Admitted patient care & $£ 3.6$ \\
A\&E visits & $£ 13.6$ \\
OTC colic medicines & $£ 9.6$ \\
OTC antiregurgitation formulas & $£ 72.3$ \\
\hline Total costs &
\end{tabular}

A\&E, accident and emergency; FGID, functional gastrointestinal disorder; GP, general practitioner; OTC, over the counter.

\section{DISCUSSION}

There is compelling evidence of discrepancies between the guidelines for the diagnosis and treatment of FGIDs, what physicians recommend and what parents may do. Our systematic literature review reports a multitude of different treatments and approaches to manage infant FGIDs that are used or have been trialled. Those reported interventions may still represent only a fraction of the remedies that are being used on a daily basis. It is outside the scope of this review to evaluate the efficacy of any intervention mentioned here, although for some OTC remedies, it appears that tolerance and safety data from clinical studies are lacking.

We hypothesised that the management of FGIDs is associated with considerable expense and, in the absence of any complete COI dataset identified in the systematic literature review, we chose England as the focus of a COI calculation because of the availability and quality of data on public and private healthcare resource use.

Medicines or formulas prescribed in England to infants with FGIDs are free at the point of consumption: the entire cost is borne by the NHS. The prescribed items considered in this analysis, with the number and costs of prescriptions made in England, are available from the HSCIC. The latest data available are from 2014 to 2015 and cover prescriptions made in both primary and secondary care. However, the taxpayer does not meet all the costs of healthcare in England. Most alternative therapies are not provided free of charge and medications that do not require a prescription can be purchased at a pharmacy.

Our analysis has shown that the cost of FGIDs is substantial, costing a minimum of $£ 72.3$ million in England in $2014 / 2015$ ( $£ 50$ million to the NHS). This estimate is likely to be significantly higher in reality as we have adopted a conservative approach in our estimates.

Expenditure per capita on healthcare in England is among the lowest of all developed countries. ${ }^{51}$ If this is the case for all age groups, then it would suggest that the estimate for England is at the lower end compared with expenditure in other developed countries for infants with FGIDs. Regardless, FGIDs are costly, both to parents and to the NHS in England, with substantial expenditure on treatments for which there is limited or no evidence of efficacy.

Our calculations are conservative both in the assumptions on which they are based and the costs which have been excluded. The latter include:

1. Alternative therapies.

2. Diagnostic or treatment costs for admitted infants

3. Outpatient consultations

4. PIPs.

5. Days taken off work by parents or carers (absenteeism).

6. Reduced productivity of parents at work (presenteeism).

7. Costs associated with side effects from inappropriate interventions. 
8. Prescriptions of constipation remedies such as lactulose.

9. Prescriptions and OTC purchases of antiallergy and comfort formulas for infants who actually have an FGID.

These exclusions are both a strength and a limitation of the analysis. The exclusions provide confidence that the estimated cost is a true lower bound of the actual cost, but they result in an estimate that, by design, is not the true cost. The exclusions also indicate areas where further research is required. The total cost presented here is likely to be a significant underestimate of the true cost as lower bound estimates were used where applicable, and several costs could not be adequately estimated and were omitted from the calculation. Where necessary, estimates and assumptions were chosen to provide consistently a lower bound of the potential cost.

We estimated that the total yearly cost of therapies for FGIDs in infants in England was $£ 72.3$ million excluding antiallergy formulas. Records indicate that there are approximately 700000 newborns per year. If $30 \%$ of these infants experienced FGIDs that required some kind of treatment, 210000 infants per year would be affected. Dividing the total costs per year by the number of affected infants, we estimate a cost of $£ 348$ per infant in the first year after birth.

It is likely that most of the care of infants for FGIDs is met in the primary and community setting and this is borne out by the estimates. However, our estimates about the time spent by health visitors were based on little actual data on resource use but are, we consider, conservative.

It is not possible to determine whether all OTC medications purchased were recommended by a physician, pharmacist or other HCP. It was, however, reported in another study conducted in six countries that overall $17 \%$ of the paediatric prescriptions were for herbal remedies and $15 \%$ were for homoeopathic preparations. ${ }^{52}$

In conclusion, we found that FGIDs in infants generate substantial expense for parents and the healthcare system. Our estimate is likely to be lower than the real cost because of missing data and evidence.

The number and type of products sold to treat FGIDs suggested that some physicians do not follow treatment guidelines. Some infants are being medicated unnecessarily, which is potentially detrimental to patient health outcomes and definitely a wasted cost, either to the taxpayer or to parents. This may be the consequence of parental demands, but may also be a gap on the provision of parental reassurance. These findings support the impression of those co-authors who are paediatric gastroenterologists practising in different parts of the world (CL, NT, MM, MS, SHQ, HS) who see in consultation infants with FGIDs who frequently have been treated not in accordance to guidelines.

Further research is required to understand why some physicians are choosing to medicate and what strategies could be adopted such that doctors and parents can manage symptoms by following clinical guidelines without resorting to costly remedies and treatments with limited or no evidence on their effectiveness. The potential cost savings and improved health outcomes are significant if such strategies and options could be put in place.

\section{Author affiliations}

${ }^{1}$ York Health Economics Consortium, University of York, York, UK

${ }^{2}$ Department of Pediatrics, Section of Pediatric Gastroenterology, Hepatology and Tranplantation, Hospital Italiano, Buenos Aires, Argentina

${ }^{3}$ Nutricia Research, Singapore

${ }^{4}$ Department of Pediatrics, Great Ormond Street Hospital, London, UK

${ }^{5}$ Division of Pediatric Gastroentrology, Hepatology and Nutrition, Sheikh Khalifa

Medical City, Abu Dhabi, United Arab Emirates

${ }^{6}$ Department of Pediatrics, Nationwide Children's Hospital, Ohio, USA

${ }^{7}$ Department of Pediatrics, National University of Singapore, Singapore

${ }^{8}$ University of Utrecht, Utrecht, The Netherlands

${ }^{9}$ Department of Pediatrics, The Medical University of Warsaw, Warsaw, Poland

Acknowledgements The authors would like to thank Dr Sarah King (record selection and data extraction of records for the systematic review), Anita Fitzgerald (systematic review report) and Dr Chris Marshall (record selection and data extraction of records for the systematic review) for their support. This work was carried out by York Health Economics Consortium, an independent consultancy.

Contributors All authors gave input on the design and aim of the systematic review. HW, JG and TL: designed the search strategy. CL, HS, ILW, MM, MS and QSH: gave input to the searchstrategy and the inclusion and exclusion criteria. JG and JM: defined the data extraction elements. JM, JG, ME and TL: wrote the protocol. $\mathrm{CL}, \mathrm{JM}, \mathrm{JMG}, \mathrm{ME}$ andTL: wrote this manuscript. CL, HS, IL-W, MM, MS, NT and QSH: revised the protocol and this manuscript. JM and JG: developed the systematic review protocol.

Funding This work was carried out by York Health Economics Consortium, an independent consultancy, and was funded by Nutricia Research, Utrecht, The Netherlands.

Competing interests TL is an employee of Nutricia Research. ILW is an employee of Danone SA. HW, JM, JG and ME are employees of YHEC. HS reports no conflicts of interest for this piece of work.CL, HS, MM, NT and QSH have served as consultants, advisory board members and/or speakers for companies manufacturing infant formulas, foods and probiotics or prebiotics. MS has served as a consultant for a medical food company.

Provenance and peer review Not commissioned; externally peer reviewed. Data sharing statement № additional data available.

Open Access This is an Open Access article distributed in accordance with the Creative Commons Attribution Non Commercial (CC BY-NC 4.0) license, which permits others to distribute, remix, adapt, build upon this work non-commercially, and license their derivative works on different terms, provided the original work is properly cited and the use is non-commercial. See: http://creativecommons.org/ licenses/by-nc/4.0/

(c) Article author(s) (or their employer(s) unless otherwise stated in the text of the article) 2017. All rights reserved. No commercial use is permitted unless otherwise expressly granted.

\section{REFERENCES}

1. Benninga MA, Faure C, Hyman PE, et al. Childhood functional gastrointestinal disorders: Neonate/toddler. Gastroenterology 2016;150:1443-55.

2. lacono G, Merolla R, D'Amico D, et al. Gastrointestinal symptoms in infancy: a population-based prospective study. Dig Liver Dis $2005 ; 37: 432-8$

3. Vandenplas Y, Abkari A, Bellaiche M, et al. Prevalence and Health Outcomes of Functional Gastrointestinal Symptoms in Infants From Birth to 12 Months of Age. J Pediatr Gastroenterol Nutr 2015;61:531-7.

4. Vandenplas $\mathrm{Y}$, Benninga M, Broekaert I, et al. Functional gastro-intestinal disorder algorithms focus on early recognition, parental reassurance and nutritional strategies. Acta Paediatr 2016;105:244-52. 
5. Hyman PE, Milla PJ, Benninga MA, et al. Childhood functional gastrointestinal disorders: neonate/toddler. Gastroenterology 2006;130:1519-26.

6. Korterink JJ, Ockeloen L, Benninga MA, et al. Probiotics for childhood functional gastrointestinal disorders: a systematic review and meta-analysis. Acta Paediatr 2014;103:365-72.

7. Headley J, Northstone K. Medication administered to children from 0 to 7.5 years in the Avon Longitudinal Study of Parents and Children (ALSPAC). Eur J Clin Pharmacol 2007;63:189-95.

8. Glanville J, Ludwig T, Lifschitz $\mathrm{C}$, et al. Costs associated with functional gastrointestinal disorders and related signs and symptoms in infants: a systematic review protocol. BMJ Open 2016;6:e011475.

9. Gieruszczak-Białek D, Konarska Z, Skórka A, et al. No effect of proton pump inhibitors on crying and irritability in infants: systematic review of randomized controlled trials. J Pediatr 2015;166:767-70.

10. Jackson MA, Goodrich JK, Maxan ME, et al. Proton pump inhibitors alter the composition of the gut microbiota. Gut 2016;65:749-56.

11. Cohen S, Bueno de Mesquita M, Mimouni FB. Adverse effects reported in the use of gastroesophageal reflux disease treatments in children: a 10 years literature review. Br J Clin Pharmacol 2015;80:200-8.

12. Aviner $\mathrm{S}$, Berkovitch $\mathrm{M}$, Dalkian $\mathrm{H}$, et al. Use of a homeopathic preparation for "infantile colic" and an apparent life-threatening event. Pediatrics 2010;125:e318-e323.

13. Lucassen PL, Assendelft WJ, van Eijk JT, et al. Systematic review of the occurrence of infantile colic in the community. Arch Dis Child 2001;84:398-403.

14. De Montfort University. Development and preliminary evaluation of an intervention package to support parents of excessively crying infants. Identifier: ISRCTN84975637. ISRCTN Registry [Internet]. London: BioMed Central, 2014. http://www.isrctn.com/ISRCTN84975637.

15. Sethi S, Mikami S, Leclair J, et al. Inpatient burden of constipation in the United States: an analysis of national trends in the United States from 1997 to 2010. Am J Gastroenterol 2014;109:250-6.

16. Sommers T, Corban C, Sengupta N, et al. Emergency department burden of constipation in the United States from 2006 to 2011. Am J Gastroenterol 2015;110:572-9.

17. Park R, Mikami S, LeClair J, et al. Inpatient burden of childhood functional GI disorders in the USA: an analysis of national trends in the USA from 1997 to 2009. Neurogastroenterol Motil 2015;27:684-92.

18. Dupont C, Rivero M, Grillon C, et al. Alpha-lactalbumin-enriched and probiotic-supplemented infant formula in infants with colic: growth and gastrointestinal tolerance. Eur J Clin Nutr 2010;64:765-7.

19. Coccorullo $\mathrm{P}$, Strisciuglio $\mathrm{C}$, Martinelli M, et al. Lactobacillus reuteri (DSM 17938) in infants with functional chronic constipation: a double-blind, randomized, placebo-controlled study. J Pediatr 2010;157:598-602.

20. Savino F, Ceratto S, Poggi E, et al. Preventive effects of oral probiotic on infantile colic: a prospective, randomised, blinded, controlled trial using Lactobacillus reuteri DSM 17938. Benef Microbes 2015;6:245-51.

21. Savino F, Cordisco L, Tarasco V, et al. Lactobacillus reuteri DSM 17938 in infantile colic: a randomized, double-blind, placebocontrolled trial. Pediatrics 2010;126:e526-e533.

22. Savino F, Palumeri E, Castagno E, et al. Reduction of crying episodes owing to infantile colic: A randomized controlled study on the efficacy of a new infant formula. Eur J Clin Nutr 2006;60:1304-10.

23. Savino F, Pelle E, Palumeri E, et al. Lactobacillus reuteri (American Type Culture Collection Strain 55730) versus simethicone in the treatment of infantile colic: a prospective randomized study. Pediatrics 2007;119:e124-130.

24. Skjeie $H$, Skonnord T, Fetveit $A$, et al. Acupuncture for infantile colic: a blinding-validated, randomized controlled multicentre trial in general practice. Scand J Prim Health Care 2013;31:190-6.

25. Szajewska H, Gyrczuk E, Horvath A. Lactobacillus reuteri DSM 17938 for the management of infantile colic in breastfed infants: a randomized, double-blind, placebo-controlled trial. J Pediatr 2013:162:257-62.

26. Infante Pina D, Badia Llach X, Ariño-Armengol B, et al. Prevalence and dietetic management of mild gastrointestinal disorders in milkfed infants. World J Gastroenterol 2008;14:248-54.

27. Landgren K, Kvorning N, Hallström I. Acupuncture reduces crying in infants with infantile colic: a randomised, controlled, blind clinical study. Acupunct Med 2010;28:174-9.
28. Reinthal M, Andersson S, Gustafsson M, et al. Effects of minimal acupuncture in children with infantile colic - a prospective, quasi-randomised single blind controlled trial. Acupunct Med 2008;26:171-82.

29. Bongers ME, de Lorijn F, Reitsma JB, et al. The clinical effect of a new infant formula in term infants with constipation: a double-blind, randomized cross-over trial. Nutr J 2007;6:8.

30. Browning M, Miller J. Comparison of the short-term effects of chiropractic spinal manipulation and occipito-sacral decompression in the treatment of infant colic: A single-blinded, randomised, comparison trial. Clinical Chiropractic 2008;11:122-9.

31. Hayden C, Mullinger B. A preliminary assessment of the impact of cranial osteopathy for the relief of infantile colic. Complement Ther Clin Pract 2006;12:83-90.

32. Miller J, Newell D. Prognostic significance of subgroup classification for infant patients with crying disorders: A prospective cohort study. $J$ Can Chiropr Assoc 2012;56:40-8.

33. Salisbury AL, High P, Twomey JE, et al. A randomized control trial of integrated care for families managing infant colic. Infant Ment Health J 2012;33:110-22.

34. Keefe MR, Lobo ML, Froese-Fretz A, et al. Effectiveness of an intervention for colic. Clin Pediatr 2006;45:123-33.

35. Cirgin Ellett ML, Perkins SM. Examination of the effect of Dr. Brown's Natural Flow Baby Bottles on infant colic. Gastroenterol Nurs 2006;29:226-31.

36. Berseth $\mathrm{CL}$, Johnston $\mathrm{WH}$, Stolz SI, et al. Clinical response to 2 commonly used switch formulas occurs within 1 day. Clin Pediatr 2009;48:58-65.

37. Hill DJ, Roy N, Heine RG, et al. Effect of a low-allergen maternal diet on colic among breastfed infants: a randomized, controlled trial. Pediatrics 2005;116:e709-715.

38. Kianifar H, Ahanchian H, Grover Z, et al. Synbiotic in the management of infantile colic: a randomised controlled trial. J Paediatr Child Health 2014;50:801-5.

39. Sung V, Hiscock $\mathrm{H}$, Tang ML, et al. Treating infant colic with the probiotic Lactobacillus reuteri: double blind, placebo controlled randomised trial. BMJ 2014;348:g2107.

40. Ciftçi EK, Arikan D. Methods used to eliminate colic in infants in the eastern parts of Turkey. Public Health Nurs 2007;24:503-10.

41. Arikan D, Alp H, Gözüm S, et al. Effectiveness of massage, sucrose solution, herbal tea or hydrolysed formula in the treatment of infantile colic. J Clin Nurs 2008;17:1754-61.

42. Akçam M, Yilmaz A. Oral hypertonic glucose solution in the treatment of infantile colic. Pediatr Int 2006;48:125-7.

43. Alves JG, de Brito RC, Cavalcanti TS. Effectiveness of mentha piperita in the treatment of infantile colic: A crossover study. Evid Based Complement Alternat Med 2012;2012:1-4.

44. Chau K, Lau E, Greenberg S, et al. Probiotics for infantile colic: a randomized, double-blind, placebo-controlled trial investigating Lactobacillus reuteri DSM 17938. J Pediatr 2015;166:74-8.

45. Mi GL, Zhao L, Qiao DD, et al. Effectiveness of Lactobacillus reuteri in infantile colic and colicky induced maternal depression: a prospective single blind randomized trial. Antonie Van Leeuwenhoek 2015;107:1547-53.

46. Moravej $\mathrm{H}$, Imanieh $\mathrm{MH}$, Kashef $\mathrm{S}$, et al. Predictive value of the cow's milk skin prick test in infantile colic. Ann Saudi Med 2010;30:468-70.

47. Oshikoya KA, Senbanjo IO, Njokanma OF. Self-medication for infants with colic in Lagos, Nigeria. BMC Pediatr 2009;9:9.

48. Curtis L, Burns A. Unit costs of health and social care. Canterbury: Personal Social Services Research Unit (PSSRU), University of Kent, 2015.

49. Department of Health. NHS reference costs 2014 to 2015. London: Government Digital Service, 2015. https://www.gov.uk/government/ publications/nhs-reference-costs-2014-to-2015. (accessed 24 Aug 2017).

50. NHS Digital. Hospital Episode Statistics, Admitted Patient Care England, 2014-15 [webpage]. Leeds: NHS Digital, 2015. http://digital. nhs.uk/catalogue/PUB19124. (accessed 24 Aug 2017).

51. The King's Fund. How does nhs spending compare with health spending internationally. London: The King's Fund, 2016. http://www. kingsfund.org.uk/projects/nhs-in-a-nutshell/health-care-spendingcompared. (accessed 24 Aug 2017).

52. Beer AM, Burlaka I, Buskin S, et al. Usage and attitudes towards natural remedies and homeopathy in general pediatrics: a crosscountry overview. Glob Pediatr Health 2016;3:2333794X1562540-9. 